POLLACK PERIODICA

An International Journal for Engineering and Information Sciences

DOI: $10.1556 / 606.2016 .11 .2 .3$

Vol. 11, No. 2, pp. 29-42 (2016)

www.akademiai.com

\title{
NUMERICAL ANALYSIS OF SHORT LINK STEEL ECCENTRICALLY BRACED FRAMES UNDER SEISMIC ACTION
}

\author{
${ }^{1}$ Adina VĂTĂMAN, ${ }^{2}$ Adrian CIUTINA, ${ }^{3,4}$ Daniel GRECEA \\ 1,2,3 Department of Steel Structures and Structural Mechanics, Faculty of Civil Engineering \\ Politehnica University of Timişoara, Ioan Curea Street nr. 1, 300224 Timişoara, Romania \\ ${ }^{4}$ Romanian Academy, Timisoara Branch, Bvd. Mihai Viteazu nr. 24, 300223 Timişoara \\ Romania, e-mail: ${ }^{1}$ adina.vataman@ upt.ro, ${ }^{2}$ adrian.ciutina@upt.ro, ${ }^{3}$ daniel.grecea@upt.ro
}

Received 1 January 2016; accepted 13 April 2016

\begin{abstract}
Eccentrically braced frames are well known for their capacity of dissipating seismic energy by plastic hinge formation in the so called 'link elements', which represent the dissipative devices of the eccentrically braced frame. The link element can be short, which means it will be mainly subjected to shear forces; long being mainly subjected to bending moment or intermediate in length subjected to a combined action of shear force-bending moment. The current study is focused on the calibration of existing experimental results with numerical finite element models of steel eccentrically braced frames with short link elements and a subsequent parametrical study considering different link lengths and multiple stiffeners along the link web panel.
\end{abstract}

Keywords: Steel, Eccentrically braced frame, Short link elements, Finite element, Numerical analysis

\section{Introduction}

Steel frames subjected to seismic loading are usually designed for stiffness, in order to limit the lateral deformations. As Vayas has demonstrated in his research the deformations of non-structural elements is a concern in the serviceability limit state, but for the ultimate limit state the deformations must be limited in order to avoid instabilities due to second order effects [1]. Besides stiffness, another very important factor is seismic design is the overall ductility, as described by Gioncu [2]. A measure of the ductility is the plastic rotation capacity of the member sections. 
Eccentrically Braced Frames (EBF) are structures designed to withstand horizontal forces by axial load in the frame elements and to dissipate the energy from these forces through the dissipative mechanism. The intentional eccentricities (named link elements) in the layout of the EBF beams are designed in order to allow the yield in shear or bending of the link element. The plastic mechanism is mainly dependent on the length of the dissipative link element, distinguishing between short links (with length $e<1.6 M_{p l} / V_{p l} \mathrm{~mm}$ according to Eurocode 8 [3]), long links (length $e>3 M_{p l} / V_{p l} \mathrm{~mm}$ ) and intermediate links. The short links have a stiff behavior and exhibit the plastic energy by shear deformations of the web panel. In case of long elements, the plastic hinges are formed in bending at each site of the links while in intermediate cases the combined shear and bending will govern the plastic behavior.

The other frame members should be designed considering over-strength in order to ensure high elastic lateral stiffness of the EBF and also a good dissipation capacity through plastic hinge formation in the link element [4]. EBF structures are characterized in design by high values of the behavior factor ( $q$ greater than 6 in high ductility structures).

Modern approaches of link elements consider fixed elements (welded connection to the beam) and removable links (bolted end-plate connection on both ends of the link element). Previous research by Ioan et al. [5] has proven that bolted links are a good solution for seismic design proving good dissipation capacity of links and the added possibility of removal and replacement of the deformed elements, acting in this case as structural fuses.

Other research on the subject, undertaken by Danku et al. [6] proved through experimental testing the high values of distortion achieved by short link elements in EBFs, both in monotonic and cyclic loading conditions.

Another important and very relevant research by Ohsaki [7] revealed the possibility of optimizing the plastic energy dissipation of links in EBFs by adjusting the geometric properties and disposition of link stiffeners. In the same study Ohsaki underlines the importance and benefits of finite element software in terms of time and cost effectiveness.

Ioan and Dima [8] have conducted a study, concerning vertical links, analyzing the influence of material properties on the seismic response, with partial conclusions showing a good behavior of the vertical shear links in case of behavior factors values close to 6 .

In a first part of the current study the experimental background will be presented, followed by the calibration of the numerical models of two reference EBF specimens subjected to monotonic and cyclic loading. Finally, a parametric monotonic study concerning different link lengths, as well as the presence of multiple stiffeners along the link length will be presented.

\section{Experimental background}

The current numerical approach is based on a large experimental program that was performed at the Research Centre for Mechanics of Materials and Structural Safety (CEMSIG) of the Politehnica University of Timişoara regarding the behavior of 
eccentrically braced frames in seismic areas. The entire program included 8 full scale EBF tests that were tested both monotonically and cyclically whilst taking into consideration the difference between pure steel frames compared to a frame with a composite beam solution. The sub-assembly frame chosen for experimental testing was part of a larger structure designed as a dual frame, (Moment Resisting Frame + Eccentrically Braced Frame $=\mathrm{MRF}+\mathrm{EBF}$ ) with five storeys and three bays (outer bays of $6.5 \mathrm{~m}$ and an inner bay of $4.5 \mathrm{~m}$ ) and represented the ground floor center bay, as can be seen in Fig. 1. The dissipative link element has a length of $300 \mathrm{~mm}$ and is classified according to Eurocode 8 as short link element and consequently subjected to shear forces.

The structural design was done for the climatic and seismic conditions of the city of Bucharest. Accordingly, the peak ground acceleration $a_{g}=0.24 \mathrm{~g}$ and the control period $T c=1.6 \mathrm{~s}$. The permanent and live loads were considered as $4 \mathrm{kN} / \mathrm{m}^{2}$ and $3 \mathrm{kN} / \mathrm{m}^{2}$ respectively.

a)

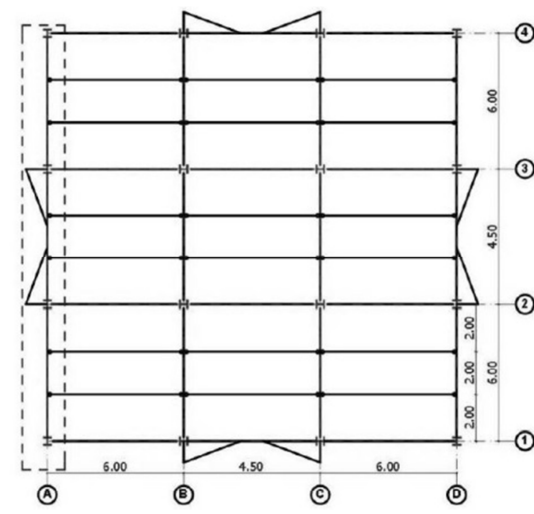

b)

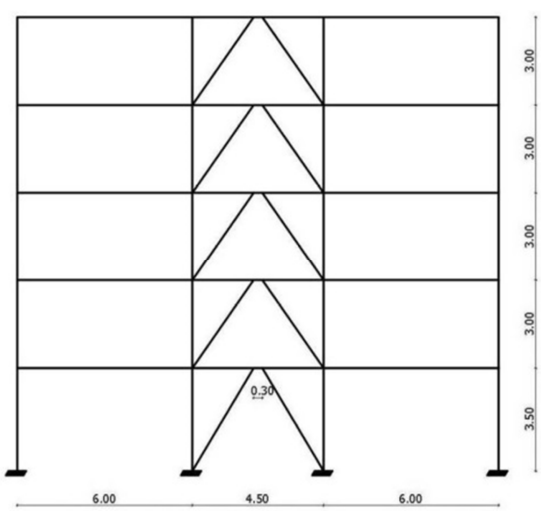

Fig. 1. Dual frame structure, a) Plane view and b) lateral view

The optimum design conducted to a steel profile HE200A (S235 steel grade) for the beam (including the dissipative link), the column steel profile HE260B and the braces HE180A profiles all in S355 steel grade.

The experimental program included both monotonic and cyclic loaded specimens. The lateral was applied in both cases at the top of the frame. The deformations and displacements were monitored by displacement transducers. Fig. 2 shows the testing set-up.

The current study is based on the responses of pure steel eccentrically braced frames loaded monotonic (specimen EBF-LF-M) and respectively cyclic (specimen EBF-LFC). These specimens were considered as reference specimens for the calibration of numerical EBF models. The specimen names included the series (EBF), typology with Fixed Link (LF) and type of loading Monotonic (M) or Cyclic (C).

Table I offers the mechanical characteristics of steel elements considered in the analyses. They are further used as input in the numerical models. 

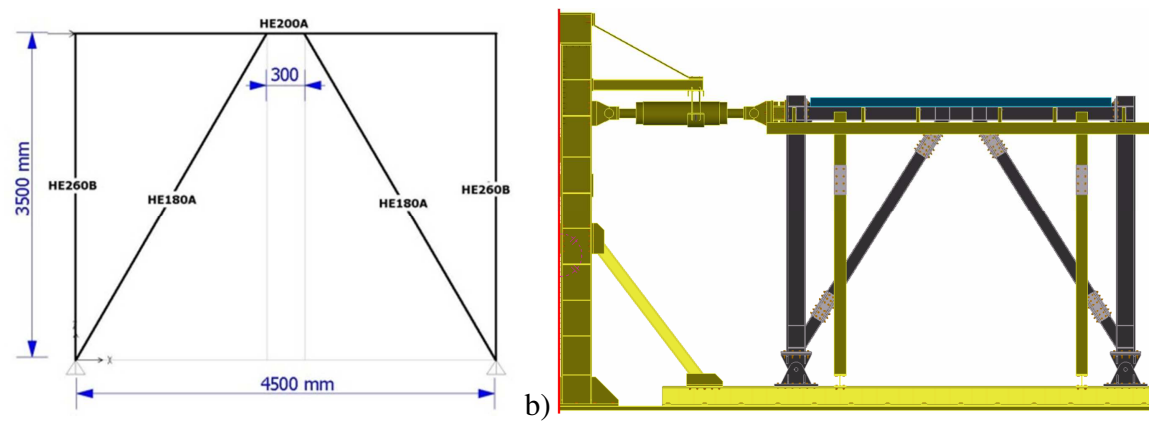

Fig. 2. a) Static scheme, b) Experimental set-up

Table I

Sectional and material properties

\begin{tabular}{|c|c|c|c|}
\hline \multicolumn{2}{|c|}{ Element profile } & Measured Steel Yield Strength & Tensile Strain at Break \\
\cline { 3 - 4 } & {$\left[\mathrm{N} / \mathrm{mm}^{2}\right]$} & {$[\%]$} \\
\hline Beam web & HE200A & 304 & 32.24 \\
Beam flange & HE200A & 323 & 35.01 \\
Column & HE260B & 355 & 15 \\
Brace & HE180A & 355 & 15 \\
\hline
\end{tabular}

The monotonic loaded specimen (EBF-LF-M) has shown a very ductile behaviour with good dissipation capacity of the link element with high values of distortion up to $250 \mathrm{mrad}$ corresponding to a maximum displacement of $180 \mathrm{~mm}$ at the top of the frame. The maximum resistance was of $550 \mathrm{kN}$, as can be observed in Fig. 3. It is to be mentioned that the high value of distortion of $250 \mathrm{mrad}$ is about four times higher than the one mentioned in the norm of $80 \mathrm{mrad}$. The failure mode was by pure shear of the link element and the ending of testing was decided due to the limitation of the actuator's stroke and not due to the physical deterioration of the specimen (see Fig. 6d).

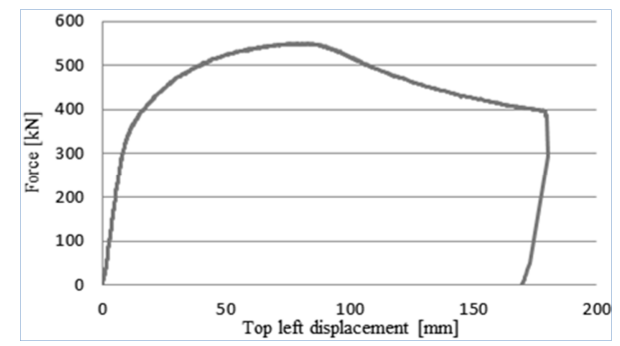

a)

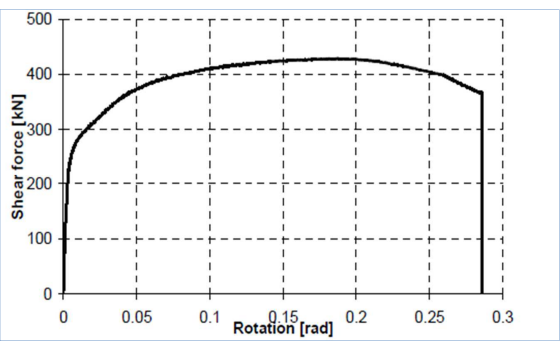

b)

Fig. 3. EBF-LF-M response, a) Force-displacement curve, b) Shear force-rotation curve 
The similar frame subjected to cyclic loading - EBF-LF-C, has shown also high values of ductility, with distortions exceeding $150 \mathrm{mrad}(\mathrm{Fig} .4 \mathrm{c})$. In this case the failure was due to alternate shear buckling of the link web panel in positive and negative cycles, as seen in Fig. 4a [9].

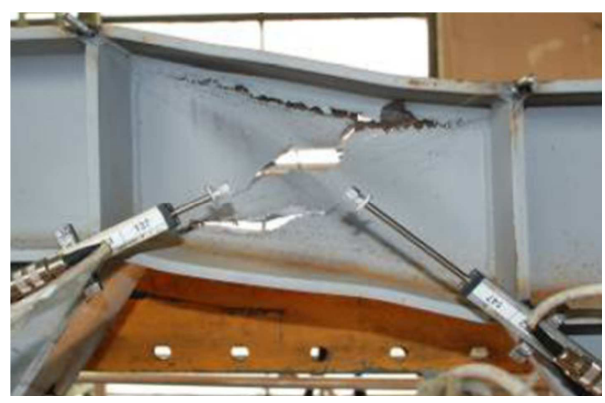

a)

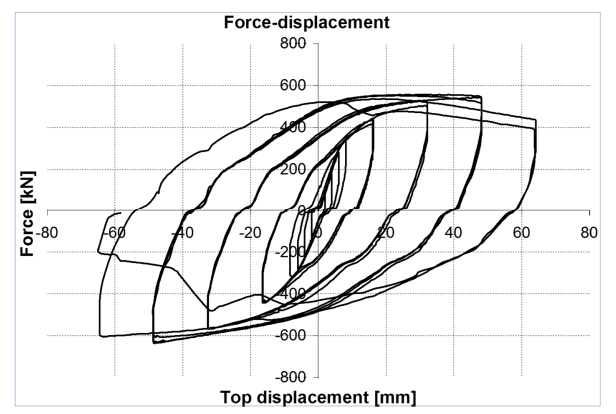

b)

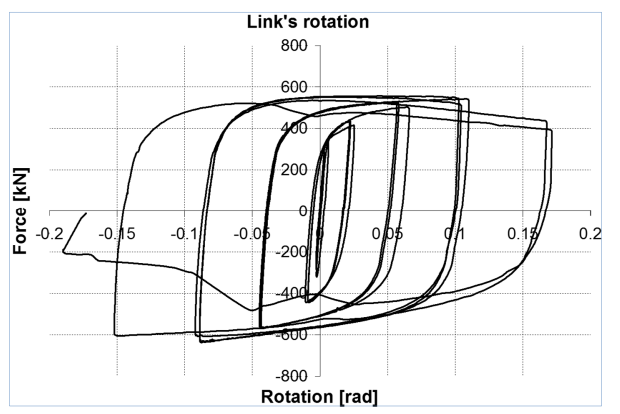

c)

Fig. 4. EBF-LF-C, a) Deformation of shear link (experimental),

b) Force-displacement curve, c) Shear force-rotation curve

\section{Numerical model and calibration}

The numerical models of the tested frames were analyzed using the finite element software Abaqus [10]. The geometrical dimensions correspond to the measured layout and cross-sectional dimensions of the reference specimen. The material characteristics simulated the results obtained by the traction tests on coupons and translated into true stress-strain values [11]. As the only area expected to reach plastic deformations is the one that contains the link element, the true stress-strain material properties are not as significant for braces and column, these are expected to perform solely in the elastic domain.

The frame members were modeled as solid finite elements with hex type elements used for meshing, considering a sweep technique with mixed medial axis and advancing front algorithms. The finite element size ranges from a fine mesh with $6 \mathrm{~mm}$ elements 
on the dissipative area of the frame (link element and adjacent beam area) to larger element sizes of $15 \mathrm{~mm}$ for braces, $20 \mathrm{~mm}$ for beam and beam stiffeners and $25 \mathrm{~mm}$ for columns and column stiffeners (Fig. 5).

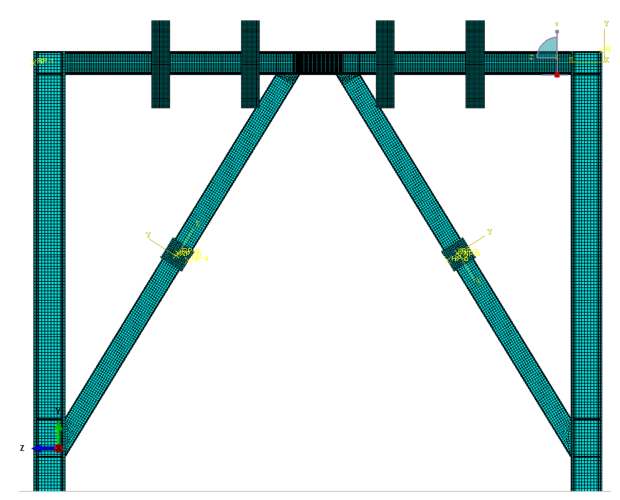

Fig. 5. EBF finite element model meshing

Due to the fact that the initial calibration curve (FEModel) has shown a stiffer behavior as compared to the experimental curve, the next step was to include in the model the additional flexibilities recorded in members and connections: the recorded slip during the experiment in the brace continuity connection was modeled using a connector and a sliding rigid body element for each brace, with a behavior law similar to the recorded one, as $4 \mathrm{~mm}$ to $500 \mathrm{kN}$ (see Fig. 6a) the FEModel+Slip).

The final plastic FE calibration was done by adjusting the lateral support clearance. The mean $2 \mathrm{~mm}$ clearance each side of the beam element measured in the testing proved to lead to an appropriate model behavior as shown in Fig. 6a) LS3-H0 curve. The error provided by this curve remains under $2 \%$ as compared to the experimental response and was further used for parametrical study.

Fig. 6 presents the calibration curves for the monotonic specimen EBF-LF-M and the load-slip input curve for the connector element in braces. Fig. 7 presents the deformed shape of the frame (Fig. 7.a) and the distorted shape of the link element as compared to the experiment (Fig. $7 b$ and Fig. 7c).

The calibration of the cyclic response was performed on a similar model to the monotonic one, by maintaining the geometry and the monotonic material properties and adjusting some parameters specific to cyclic loading. The cyclic Chaboche material model [12] was adopted with 5 back-stresses in order to model the material behavior. The plastic material properties were also changed in order to provide a combined isotropic and kinematic hardening of the steel for the dissipative link element and beam element.

The cyclic loading procedure, as given by the ECCS [13] was considered (Fig. 8) based on previous research regarding numerical cyclic analysis using finite element software [14]. According to this procedure in the elastic range three different amplitudes of $0.25 D_{y}, 0.5 D_{y}$ and $0.75 D_{y}$ should be applied while in the plastic range amplitudes of $1 D_{y}, 2 D_{y}, 4 D_{y}, 6 D_{y}$ and $8 D_{y}$ were considered to simulate the cycles of experimental 
tests. $D_{y}$ represents the yield displacement resulted from the interpretation of monotonic tests, in accordance to the ECCS procedure. The load application point remained unchanged at top of the frame while it was applied in displacement control.

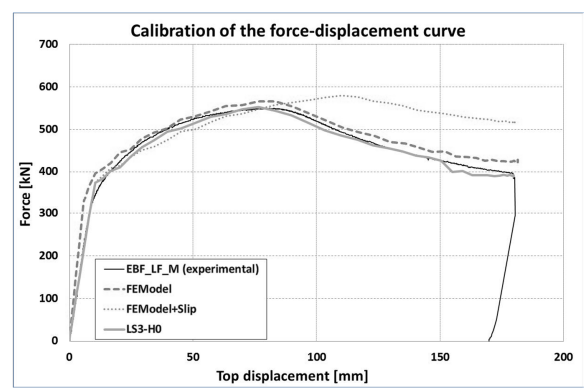

a)

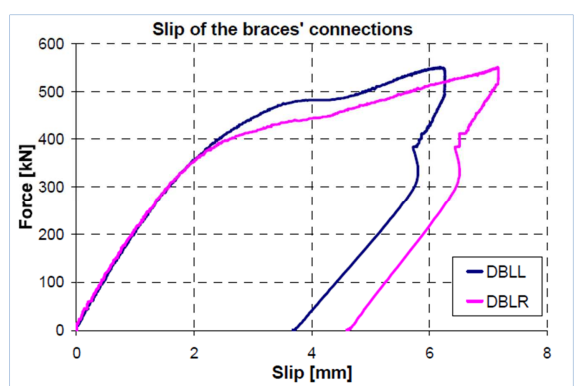

b)

Fig. 6. a) Response curves of the FE Models compared to experimental - calibration,

b) Slip in brace connections

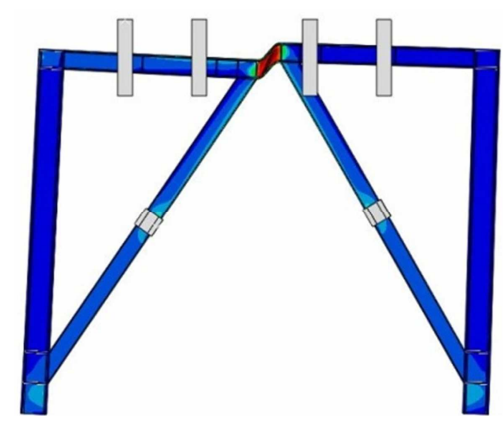

a)

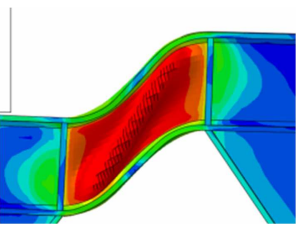

b)

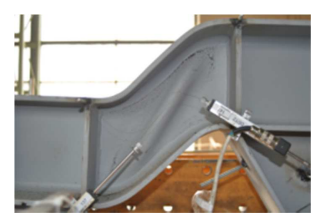

c)

Fig. 7. a) Deformed shape of calibrated EBF model, b) and c) numerical and experimental deformation of the shear link (comparison)

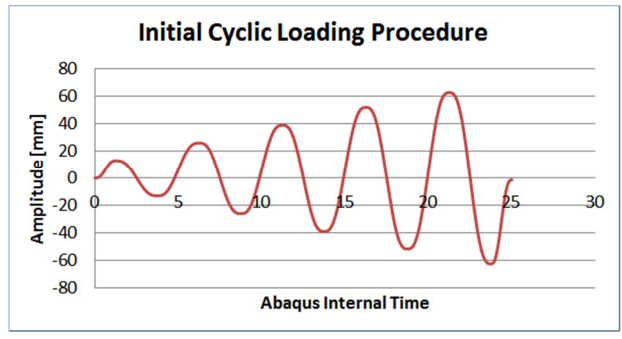

a)

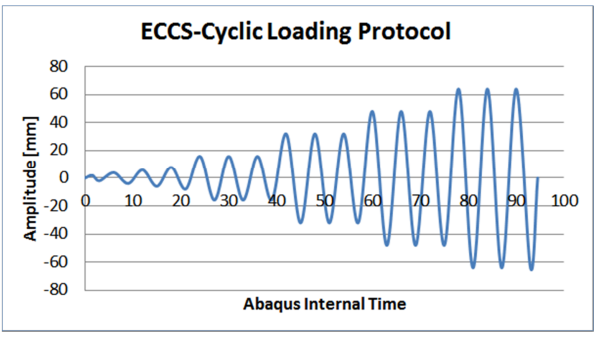

b)

Fig. 8. Cyclic loading procedure, a) Initial protocol; b) The European Convention for Constructional Steelwork (ECCS) protocol 
Another significant aspect of a cyclic analysis in FEM models is the analysis timestep, for which it was assigned a higher value compared to the corresponding monotonic one ( 25 in cyclic, 6 for monotonic). The calibration procedure was done in two steps:

- initial adjustment of cycles. This included one cycle per amplitude. This step was used to refine the overall shape of the force-displacement curve and the total analysis runtime. Special adjustments of material adjustments were done, such as passing from one to five back-stresses (see Fig. 9a);

- $\quad$ application of the entire ECCS loading protocol with three cycles per amplitude. The result of this step could be seen in Fig. $9 b$.

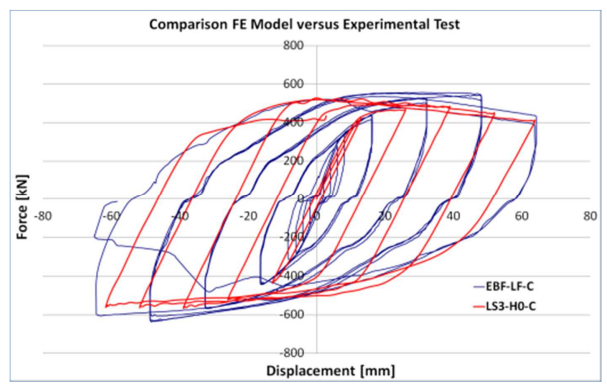

a)

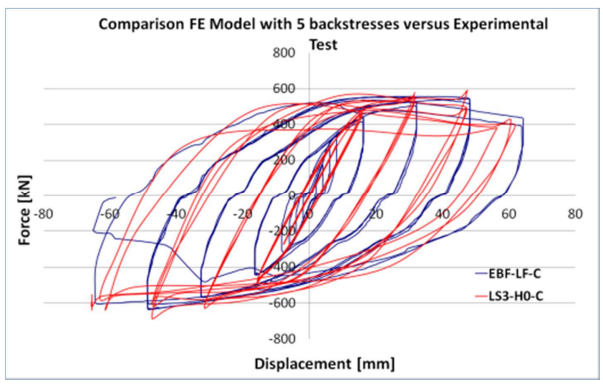

b)

Fig. 9. Calibration curves for cyclic loading

The resulting calibration curve shows very good approximation of the experimental resistance (less than $5 \%$ error) and also shown some differences in the initial and restoring rigidities.

\section{Parametric study and results}

After the calibration of the model subjected to monotonic loading a parametric study was undertaken consisting of a total of 16 numerical models analysed with the same finite element software, Abaqus. The main directions that were considered were the stiffening of the link, respectively the length of the short link. The parametric study was performed only considering monotonic loading. The notation of the EBF models reflect these two parameters: LS is the generic name of the series (standing for steel link), first number describes the length of the link, $\mathrm{H}$ describes the cross-section profile of the beam (HE200A) and the last figure indicates the number of stiffeners evenly distributed on the length of the link. The finite element models used in the parametric study have been described in Table II.

The normative requirement from the Eurocode 8 offers a method of establishing the intervals at which the intermediate stiffeners should be disposed along the link length. The distance equal to $\left(30 t_{w}-d / 5\right)$ where $t_{w}$ represents the web thickness and $d$ is the height of the cross-section of the link. The resulting distance is $155 \mathrm{~mm}$ between stiffeners for a HEA 200 profile. The influence of the link's stiffness on the behavior of 
the EBF was analyzed by creating three different configurations of web stiffening: no stiffeners (H0 models), one stiffener in the middle of the link (H1 models) and a third category with 2 stiffeners (H2 models). In the case of the link model with $750 \mathrm{~mm}$ length an additional model with 3 stiffeners was considered ( $\mathrm{H} 3$ models).

Table II

Configurations of analyzed EBF models

\begin{tabular}{|c|c|c|}
\hline Name of the model & Link length e [mm] & Number of stiffeners \\
\hline LS3-H0 & 300 & 0 \\
LS3-H1 & 300 & 1 \\
LS3-H2 & 300 & 2 \\
LS4-H0 & 400 & 0 \\
LS4-H1 & 400 & 1 \\
LS4-H2 & 400 & 2 \\
LS5-H0 & 500 & 0 \\
LS5-H1 & 500 & 1 \\
LS5-H2 & 500 & 2 \\
LS6-H0 & 600 & 0 \\
LS6-H1 & 600 & 1 \\
LS6-H2 & 600 & 2 \\
LS7.5-H0 & 750 & 0 \\
LS7.5-H1 & 750 & 1 \\
LS7.5-H2 & 750 & 2 \\
LS7.5-H3 & 750 & 3 \\
\hline
\end{tabular}

The second parameter considered in analyses was the length of the dissipative link element. The following lengths were chosen starting with the length from the experimental test $(300 \mathrm{~mm})$ and with respect to the normative limitations for a short dissipative link: 300 (LS3 models), 400 (LS4 models), 500 (LS5 models), 600 (LS6 models) and $750 \mathrm{~mm}$ (LS7.5 models) respectively. The maximum admissible length for short links for the HEA 200 profile is $760 \mathrm{~mm}$.

The results from the numerical simulations were evaluated by considering $V-\gamma$ (shear force - link panel distortion) response curves.

Considering the influence of the number of stiffeners, Fig. 10 shows the behaviour of the different length link models in function of the number of stiffeners. The $V-\gamma$ responses revealed a higher resistance of the models with one and two stiffeners along the link length, as well as a translation of the point of maximum force to higher values of panel distortion. This difference becomes smaller with the increase in link length.

As confirmed by Fig. 11, the elastic stiffness of the frame was not affected by the increase in stiffeners disposed on the link. In return, the link length significantly influences the initial stiffness: the longer the link, the smaller the initial stiffness. Although not visible on global $V$ - $\gamma$ curves, the initial stiffnesses are different by more than 40\%: $96121 \mathrm{kN} / \mathrm{mm}$ for LS3-H2 model, $87766 \mathrm{kN} / \mathrm{mm}$ for LS4-H2, $85373 \mathrm{kN} / \mathrm{mm}$ for LS5-H2, $73898 \mathrm{kN} / \mathrm{mm}$ for LS6-H2 and just $55407 \mathrm{kN} / \mathrm{mm}$ for the LS7.5-H2 model. 

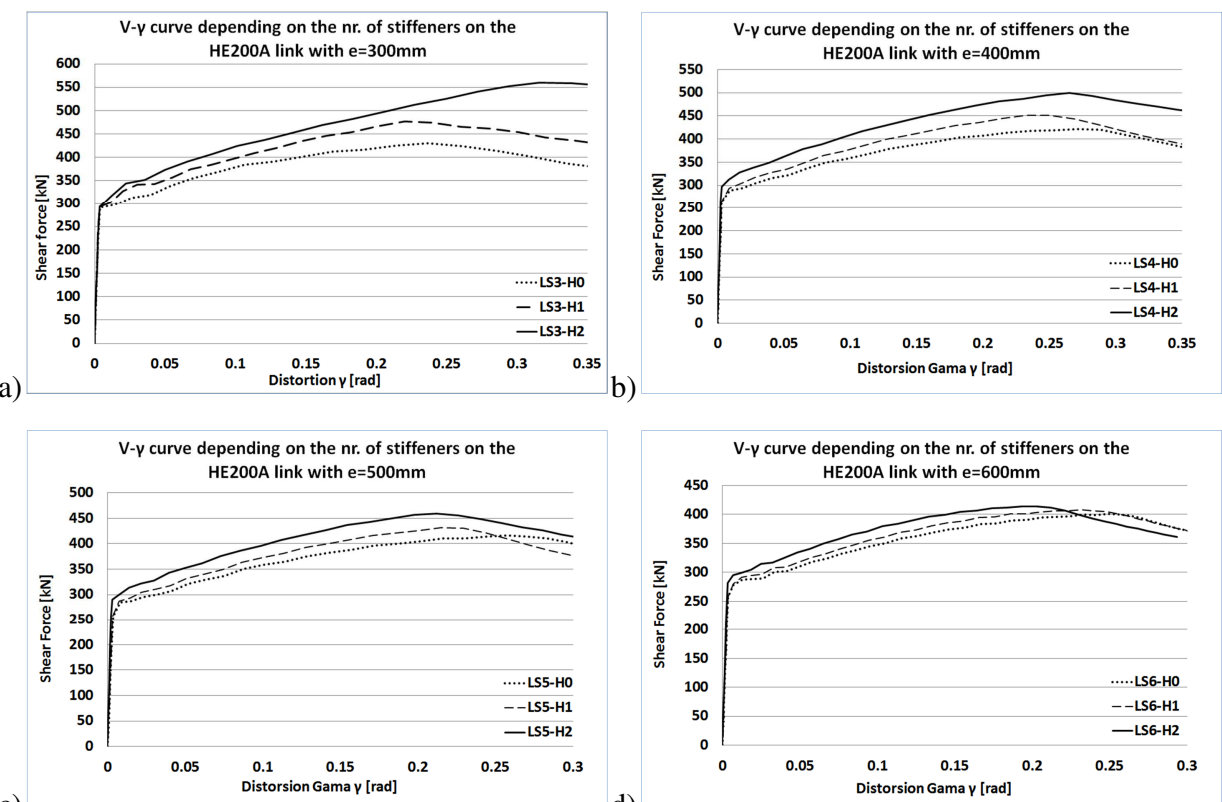

b)
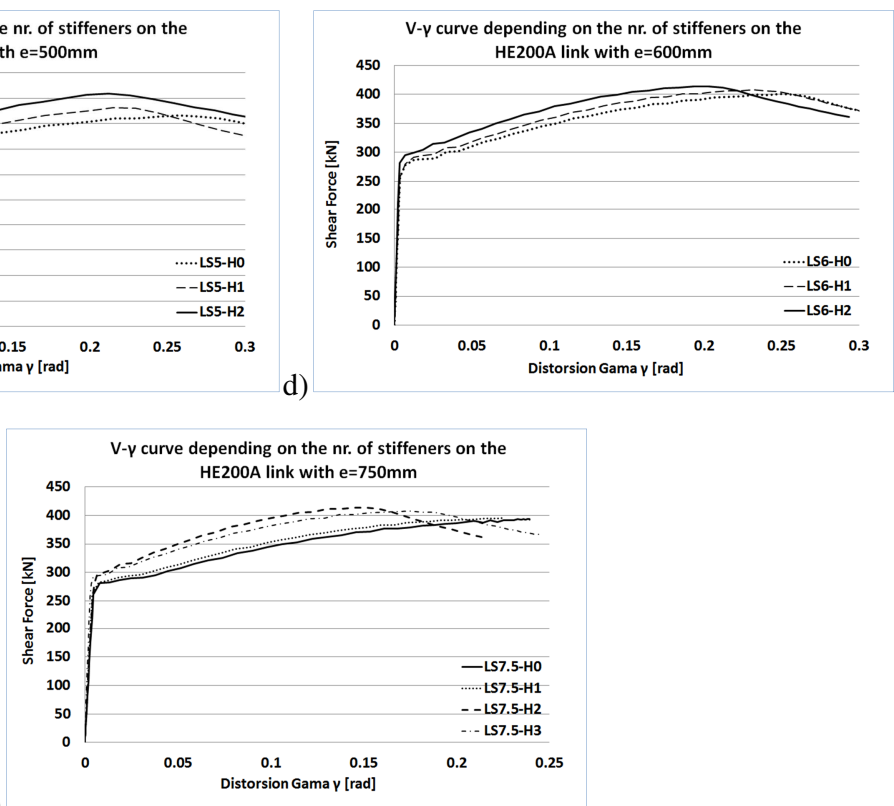

e)

Fig. 10. $V-\gamma$ response curves for different stiffened link lengths,

a) $300 \mathrm{~mm}$, b) $400 \mathrm{~mm}$, c) $500 \mathrm{~mm}$, d) $600 \mathrm{~mm}$, e) $750 \mathrm{~mm}$
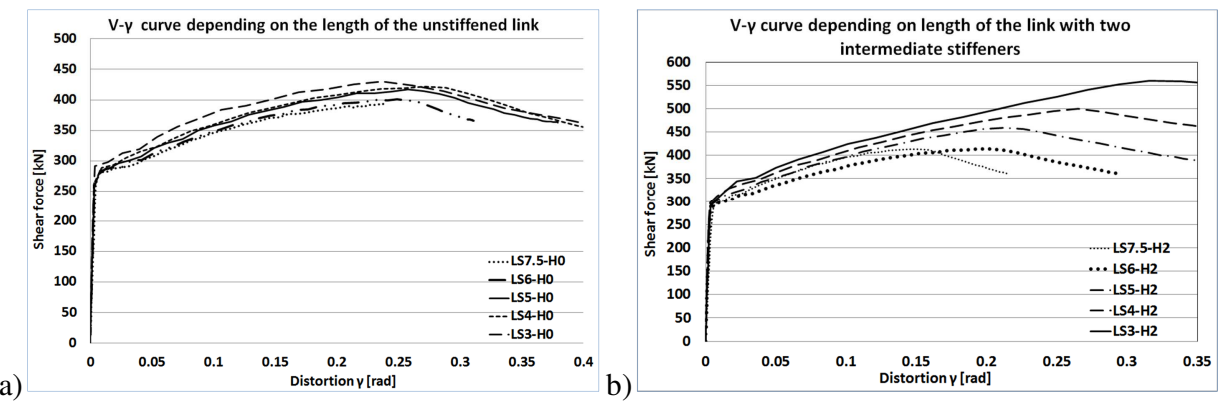

Fig. 11. $V$ - $\gamma$ response curves for different link lengths, a) unstiffened, b) with two intermediate stiffeners

Pollack Periodica 11, 2016, 2 
Regarding the influence of the link length, Fig. 11a reveals that for the unstiffened link models the length of the link has not an important influence on the overall behavior. However for the link with two intermediate stiffeners the influence becomes important (Fig. 11b). The link type with $750 \mathrm{~mm}$ length represents a particular case due to early shear buckling of the web. An additional observation must be made in this specific case, due to the proximity of this length to the limit for short-to-medium link elements $(760 \mathrm{~mm})$, hence additional stiffeners should be considered. Consequently, an additional model was created with three stiffeners along the link length, which proved a slightly smaller resistance than the previous model with two stiffeners, but higher values of distortion (Fig. 10e).

Table III presents the values of the maximum shear resistances and corresponding panel distortions for all FE models. The shorter link length model with two stiffeners provided the highest value of the highest resistance and also corresponding panel distortion, whilst the smallest values are the ones for the unstiffened $750 \mathrm{~mm}$ link. The recorded increase in resistance is of $10 \%$ for one stiffener and $30 \%$ for two stiffeners in case of $300 \mathrm{~mm}$ link model and respectively $2 \%$ and $3 \%$ in case of $600 \mathrm{~mm}$ link. These values are even lower in case of $750 \mathrm{~mm}$ link model by only $0.7 \%$ resistance increase for one stiffener, $5 \%$ for two stiffeners and a decrease of $2 \%$ for three stiffeners. The addition of another stiffener did not enhance the resistance of the shear link.

\section{Table III}

$V-\gamma$ values for the finite element EBF models

\begin{tabular}{|l|c|c|c|c|}
\hline Model & LS3-H0 & LS3-H1 & LS3-H2 & \\
\hline$V[\mathrm{kN}]$ & 430.213 & 476.283 & 559.769 & \\
$\gamma[\mathrm{rad}]$ & 0.236 & 0.220 & 0.316 & \\
\hline Model & LS4-H0 & LS4-H1 & LS4-H2 & \\
\hline$V[\mathrm{kN}]$ & 421.397 & 451.094 & 499.510 & \\
$\gamma[\mathrm{rad}]$ & 0.271 & 0.250 & 0.282 & \\
\hline Model & LS5-H0 & LS5-H1 & LS5-H2 & \\
\hline$V[\mathrm{kN}]$ & 416.730 & 432.088 & 459.440 & \\
$\gamma[\mathrm{rad}]$ & 0.258 & 0.215 & 0.226 & \\
\hline Model & LS6-H0 & LS6-H1 & LS6-H2 & \\
\hline$V[\mathrm{kN}]$ & 400.651 & 407.846 & 413.411 & \\
$\gamma[\mathrm{rad}]$ & 0.249 & 0.232 & 0.212 & \\
\hline Model & LS7.5-H0 & LS7.5-H1 & LS7.5-H2 & LS7.5-H3 \\
\hline$V[\mathrm{kN}]$ & 393.437 & 396.849 & 413.411 & 407.846 \\
$\gamma[\mathrm{rad}]$ & 0.239 & 0.236 & 0.152 & 0.181 \\
\hline
\end{tabular}

The deformed shapes of the link elements modelled with FEM software revealed the failure mode of the structure, by plastic hinge formation by panel web crippling. By adding stiffeners the initial panel is divided in several panels, which present different deformations, as can be observed in Fig. 12. This figure shows all the deformed shapes of the link elements at for the maximum induced top displacement of $180 \mathrm{~mm}$. 

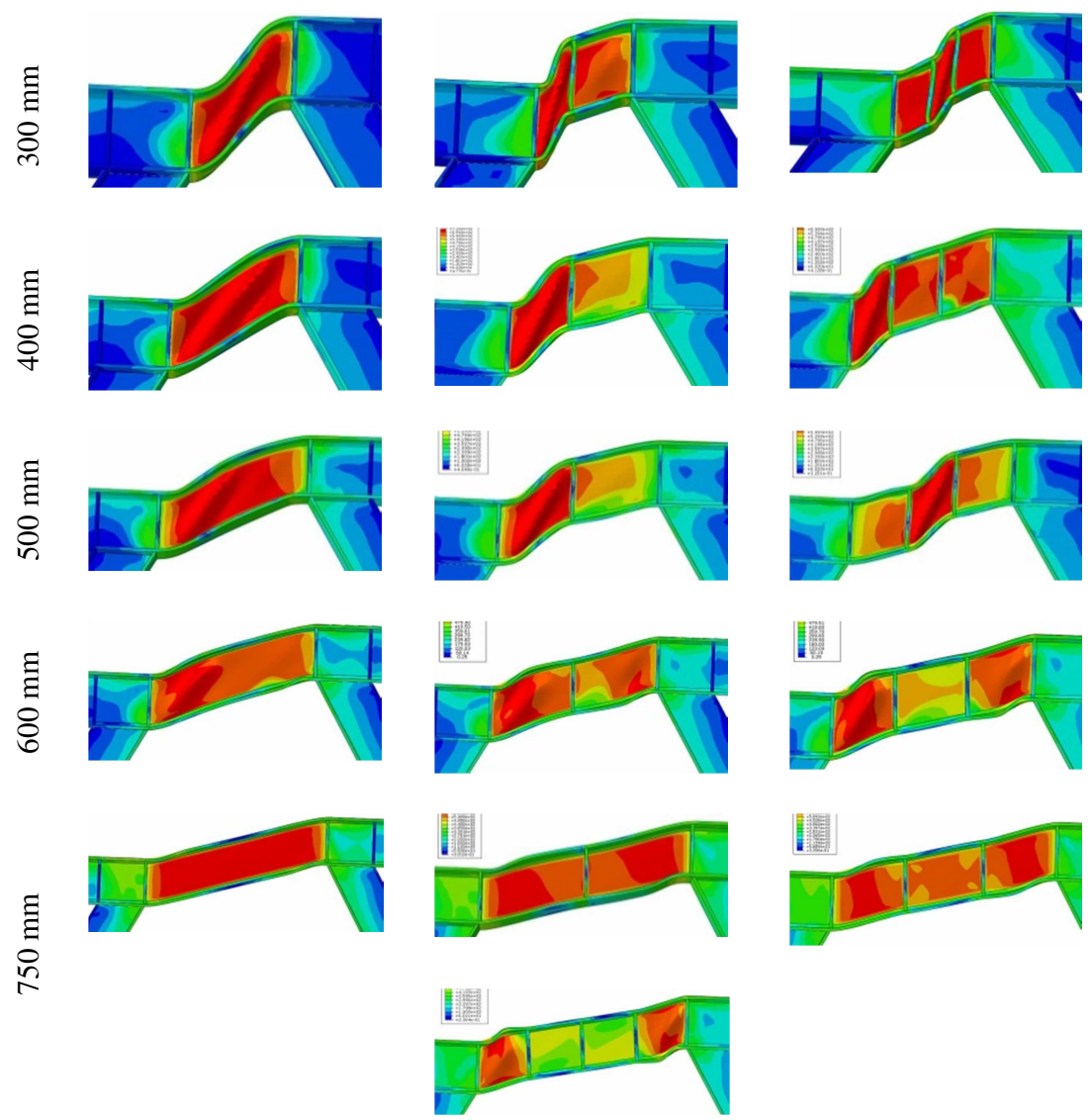

Fig. 12. Deformed link shapes related to link length and number of stiffeners

\section{Conclusions}

The main purpose of the present paper is the characterization of the behavior of link short elements in Eccentrically Braced Frames subjected to lateral loads. The study is developed by finite element models on the basis of a prior calibration on experimental tests. The following conclusions could be drawn from the study:

- both monotonic and cyclic experimental responses can be modeled by FE Models, with good accuracy but only by control of the behavior of the materials, attentive modeling of the experimental conditions such as connection slips and lateral restraining conditions;

- both experimental and FE models proved that the link elements can provide high values of panel distortions of $250 \mathrm{mrad}$ in monotonic loading and $150 \mathrm{mrad}$ in cyclic loading, much higher than the minimum values indicated in the norms; 
- $\quad$ increasing the number of intermediate stiffeners in the link geometry conduct to higher resistances and hardening stiffness, but does not play any influence in elastic behavior. However, this may share the global distortion in panels and eventually the plastic distortion will develop in one of the panels;

- long-length link elements, close to intermediate-length links can lead to early failure shear buckling and consequently at smaller resistances and global distortions.

In order to have a full over-view of the behavior of link elements subjected to shear and/or bending, the results of the FE simulations should be extended to cyclic parametrical study or other link typologies such as higher lengths, connection to the concrete or web slenderness.

\section{Acknowledgements}

This work was partially supported by the strategic grant POSDRU/159/1.5/S/137516 of the Ministry of National Education of Romania, co-financed by the European Social Fund - Investing in People, within the Sectorial Operational Programme Human Resources Development 2007-2013.

\section{References}

[1] Vayas I. Design of braced frames, In: Seismic resistant steel structures, Chapter 5, CISM Courses and Lectures, No. 420, 2000.

[2] Gioncu V., Mazzolani F. Seismic design of steel structures, CRC Press Taylor and Francis Group, 2014.

[3] EN 1998-1 EUROCODE 8, Design of structures for earthquake resistance, Part 1. General rules, seismic actions and rules for buildings, Brussels, CEN, European Committee for Standardization, 2003.

[4] Earthquake resistant steel structures, Arcelor Mittal Europe, http://sections.arcelormittal. $\mathrm{com} /$ fileadmin/redaction/4-Library/1-Sales_programme_Brochures/Earthquake/Earthquake _EN.pdf, (last visited 28 December 2015).

[5] Ioan A., Stratan A., Dubina D. Numerical simulation of bolted links removal in eccentrically braced frames, Pollack Periodica, Vol. 8, No. 1, 2013, pp. 15-26.

[6] Danku G., Ciutina A., Dubina D. Influence of steel-concrete interaction in dissipative zones of frames: II, Numerical study, Steel and Composite Structures, Vol. 15, No. 3, 2013, pp. 323-348.

[7] Ohsaki, M., Nakajima, T. Optimization of link member of eccentrically braced frames for maximum energy dissipation, Journal of Constructional Steel Research, Vol. 75, 2012, pp. 38-44.

[8] Ioan P., Dima S. Behavior of eccentrically braced structures having active links connected or not with R.C. slab, The $9^{\text {th }}$ Nordic Steel Construction Conference, Helsinki, Finland, 1820 June 2001, pp. 679-688.

[9] Danku G. Study of the development of plastic hinges in composite steel-concrete structural members subjected to shear and/or bending, PhD Thesis, Politehnica University of Timisoara, 2011.

[10] Abaqus 6.11-1, Dassault Systèmes Simulia Corp, 2011. 
[11] Arasaratnam P., Sivakumaran K. S., Tait M. J. True stress-strain models for structural steel elements, International Scholarly Research Notices (ISRN) Civil Engineering, Vol. 2011, Article ID 656401

[12] Chaboche J. L. A review of some plasticity and viscoplasticity constitutive theories, International Journal of Plasticity Vol. 24, No. 10, 2008, pp. 1642-1693.

[13] European Convention for Constructional Steelwork, ECCS TWS 1.3 N.45/86, Recommended testing procedure for assessing the behavior of structural steel elements under cyclic loads, 1986.

[14] Vulcu C., Stratan A., Dubina D. Numerical simulation of the cyclic loading for welded beam-to-cft- column joints of dual-steel frames, Pollack Periodica, Vol. 7, No. 2, 2012, pp. 35-46. 